\title{
FROBENIUS VECTORS, HILBERT SERIES AND GLUINGS OF AFFINE SEMIGROUPS
}

\author{
A. ASSI, P.A. GARCÍA-SÁNCHEZ AND I. OJEDA
}

\begin{abstract}
Let $S_{1}$ and $S_{2}$ be two affine semigroups, and let $S$ be the gluing of $S_{1}$ and $S_{2}$. Several invariants of $S$ are related to those of $S_{1}$ and $S_{2}$; we review some of the most important properties preserved under gluings. The aim of this paper is to prove that this is the case for the Frobenius vector and the Hilbert series. Applications to complete intersection affine semigroups are also given.
\end{abstract}

1. On gluings of affine semigroups. In this section, we briefly summarize results on the gluing of affine semigroups. We also introduce concepts and notation used throughout the paper.

An affine semigroup $S$ is a finitely generated submonoid of $\mathbb{Z}^{m}$ for some positive integer $m$. If $S \cap(-S)=0$, that is to say, $S$ is reduced, it can be shown that it has a unique minimal system of generators (see, for instance, [25, Chapter 3]). The cardinality of the minimal generating system of $S$ is known as the embedding dimension of $S$. Recall that each reduced affine semigroup can be embedded into $\mathbb{N}^{m}$ for some $m$. In the following, we will assume that our affine semigroups are submonoids of $\mathbb{N}^{m}$.

Given an affine semigroup $S \subseteq \mathbb{N}^{m}$, denote by $\mathrm{G}(S)$ the group spanned by $S$, that is,

$$
\mathrm{G}(S)=\left\{\mathbf{z} \in \mathbb{Z}^{m} \mid \mathbf{z}=\mathbf{a}-\mathbf{b}, \mathbf{a}, \mathbf{b} \in S\right\} .
$$

2010 AMS Mathematics subject classification. Primary 20M14, Secondary 05A15, 11D07, 14M10.

Keywords and phrases. Affine semigroup, gluing, Frobenius vector, Hilbert series.

The first author is partially supported by project GDR CNRS 2945. The second author is supported by projects MTM2010-15595, MTM2014-55367-P, FQM-343, FQM-5849, Géanpyl (FR n ${ }^{\circ} 2963 \mathrm{du}$ CNRS) and FEDER funds. The third author is partially supported by project MTM2012-36917-C03-01, National Plan I+D+I and by Junta de Extremadura (FEDER) funds.

Received by the editors on November 8, 2013. 
Let $A$ be the minimal generating system of $S$, and let $A=A_{1} \cup A_{2}$ be a nontrivial partition of $A$. Let $S_{i}=\left\langle A_{i}\right\rangle$ (the monoid generated by $\left.A_{i}\right), i \in\{1,2\}$. Then $S=S_{1}+S_{2}$. We say that $S$ is the gluing of $S_{1}$ and $S_{2}$ by $\mathbf{d}$ if

- $\mathbf{d} \in S_{1} \cap S_{2}$, and

- $\mathrm{G}\left(S_{1}\right) \cap \mathrm{G}\left(S_{2}\right)=\mathbf{d} \mathbb{Z}$.

We will denote this fact by $S=S_{1}+{ }_{\mathrm{d}} S_{2}$.

There are several properties that are preserved under gluings, and also some invariants of a gluing $S_{1}+_{\mathbf{d}} S_{2}$ can be computed by knowing their values in $S_{1}$ and $S_{2}$. We summarize some of them next.

Assume that $A=\left\{\mathbf{a}_{1}, \ldots, \mathbf{a}_{k}\right\}$. The monoid homomorphism $\varphi$ : $\mathbb{N}^{k} \rightarrow S$ induced by $\mathbf{e}_{i} \mapsto \mathbf{a}_{i}, i \in\{1, \ldots, k\}$, is an epimorphism (where $\mathbf{e}_{i}$ is the $i$ th row of the $k \times k$ identity matrix). Thus, $S$ is isomorphic as a monoid to $\mathbb{N}^{k} / \operatorname{ker} \varphi$, where $\operatorname{ker} \varphi$ is the kernel congruence of $\varphi$, that is, the set of pairs $(\mathbf{a}, \mathbf{b}) \in \mathbb{N}^{k} \times \mathbb{N}^{k}$ with $\varphi(\mathbf{a})=\varphi(\mathbf{b})$. A presentation of $S$ is a system of generators of $\operatorname{ker} \varphi$. A minimal presentation is a presentation such that none of its proper subsets is a presentation. All minimal presentations have the same (finite) cardinality (see, for instance, [25, Corollary 9.5]). Suppose that $S=S_{1}+_{\mathbf{d}} S_{2}$, where $S_{i}=\left\langle A_{i}\right\rangle$ for $i \in\{1,2\}$, and that $A=A_{1} \cup A_{2}$ is a nontrivial partition of $A$. We may assume, without loss of generality, that $A_{1}=\left\{\mathbf{a}_{1}, \ldots, \mathbf{a}_{l}\right\}$ and $A_{2}=\left\{\mathbf{a}_{l+1}, \ldots, \mathbf{a}_{k}\right\}$. According to [22, Theorem 1.4], if we know minimal presentations of $S_{1}$ and $S_{2}$, then we can construct a minimal presentation of $S$ in the following way. Let $\rho_{i}$ be a minimal presentation of $S_{i}, i \in\{1,2\}$. Take $(\mathbf{a}, \mathbf{b}) \in \mathbb{N}^{k} \times \mathbb{N}^{k}$ with $\varphi(\mathbf{a})=\varphi(\mathbf{b})=\mathbf{d}$, the first $l$ coordinates of $\mathbf{b}$ equal to zero and the last $k-l$ coordinates of $\mathbf{a}$ equal to zero. Then

$$
\rho=\rho_{1} \cup \rho_{2} \cup\{(\mathbf{a}, \mathbf{b})\}
$$

is a minimal presentation of $S$ (actually, [22, Theorem 1.4] asserts that this characterizes that $\left.S=S_{1}+_{\mathbf{d}} S_{2}\right)$.

For an affine semigroup $S$ define $\operatorname{Betti}(S)$ as the set of $\mathbf{s} \in S$ for which there exists $\mathbf{a}, \mathbf{b} \in \varphi^{-1}(\mathbf{s})$ such that $(\mathbf{a}, \mathbf{b})$ belongs to a minimal presentation of $S$. Theorem 10 in [14] states that

$$
\operatorname{Betti}\left(S_{1}+{ }_{\mathbf{d}} S_{2}\right)=\operatorname{Betti}\left(S_{1}\right) \cup \operatorname{Betti}\left(S_{2}\right) \cup\{\mathbf{d}\} .
$$

Since several invariants such as the catenary degree and the maximum 
of the delta sets depend on the Betti elements of $S([\mathbf{9}, \mathbf{8}]$, respectively), the computation of these invariants for $S_{1}+_{\mathrm{d}} S_{2}$ can be performed once we know their values for $S_{1}, S_{2}$ and $\mathbf{d}$ (see, for instance, [7, Corollary 4]).

Affine semigroups with a single Betti element can be characterized as a gluing of several copies of affine semigroups with empty minimal presentation (and thus isomorphic to $\mathbb{N}^{t}$ for some positive integer $t$ ) along this single Betti element ([15]).

We say that $S$ is uniquely presented if, for every two minimal presentations $\sigma$ and $\tau$ and every $(\mathbf{a}, \mathbf{b}) \in \sigma$, either $(\mathbf{a}, \mathbf{b}) \in \tau$ or $(\mathbf{b}, \mathbf{a}) \in \tau$, that is, there is a unique minimal presentation up to rearrangement of the pairs of the minimal presentation. It is known ([14, Theorem 12]) that $S_{1}+_{\mathbf{d}} S_{2}$ is uniquely presented if and only if $S_{1}$ and $S_{2}$ are uniquely presented and $\pm(\mathbf{d}-\mathbf{a}) \notin S_{1}+_{\mathbf{d}} S_{2}$ for every $\mathbf{a} \in \operatorname{Betti}\left(S_{1}\right) \cup \operatorname{Betti}\left(S_{2}\right)$.

It is well known that the cardinality of any minimal presentation of an affine semigroup is greater than or equal to its embedding dimension minus the dimension of the vector space spanned by the semigroup. An affine semigroup is a complete intersection affine semigroup if the cardinality of any of its minimal presentations attains this lower bound. It can be shown that an affine semigroup is a complete intersection if and only if it is either isomorphic to $\mathbb{N}^{t}$ for some positive integer $t$ or it is the gluing of two complete intersection affine semigroups ([12]). This result generalizes [23], which generalizes the classical result by Delorme for numerical semigroups ([11]; actually, the definition of gluing was inspired in that paper).

A numerical semigroup is a submonoid of $\mathbb{N}$ with finite complement in $\mathbb{N}$. It is easy to see that every numerical semigroup is finitely generated (see, for instance, [26, Chapter 1]), and thus every numerical semigroup is an affine semigroup. Let $S$ be a numerical semigroup. The largest integer not belonging to $S$ is known as its Frobenius number, $\mathrm{F}(S)$. By definition, $\mathrm{F}(S)+1+\mathbb{N} \subseteq S$. This is why the integer $\mathrm{F}(S)+1$ is known as the conductor of $S$. Delorme [11] shows that the conductor of a numerical semigroup, that is, a gluing, say $S_{1}+{ }_{d} S_{2}$, can be computed in terms of the conductors of $S_{1}, S_{2}$ and $d$ (we use $d$ here instead of $\mathbf{d}$ because in this setting $d$ is an integer). Thus, a formula for the Frobenius number of a numerical semigroup that is a gluing is easily 
derived (this idea is exploited in [4] to give a procedure for computing the set of all complete intersection numerical semigroups with given Frobenius number). One of the aims of this paper is to generalize this formula for affine semigroups.

Let $S$ be a numerical semigroup. An element $g \in \mathbb{Z} \backslash S$ is a pseudoFrobenius number if $g+(S \backslash\{0\}) \subseteq S$. In particular, $\mathrm{F}(S)$ is always a pseudo-Frobenius number. The cardinality of the set of pseudoFrobenius numbers is known as the (Cohen-Macaulay) type of $S, \mathrm{t}(S)$. A numerical semigroup is symmetric if its type is one (there are plenty of characterizations of this property, see for instance, [26, Chapter 3]). Delorme in his above-mentioned paper [11] also proved that a numerical semigroup that is a gluing $S_{1}+{ }_{d} S_{2}$ is symmetric if and only if $S_{1}$ and $S_{2}$ are symmetric. Nari [20, Proposition 6.6] proved that, for a numerical semigroup of the form $S_{1}+{ }_{d} S_{2}$,

$$
\mathrm{t}\left(S_{1}+{ }_{d} S_{2}\right)=\mathrm{t}\left(S_{1}\right) \mathrm{t}\left(S_{2}\right)
$$

(actually the definition of gluing for numerical semigroups is slightly different and we have to divide $S_{1}$ and $S_{2}$ by their greatest common divisors in order to get $S_{1}$ and $S_{2}$ numerical semigroups; see the paragraph after Theorem 4.3). This formula can be seen as a generalization of the fact that the gluing of symmetric numerical semigroups is again symmetric, and it also shows that

- the gluing of pseudo-symmetric numerical semigroups (the only pseudo-Frobenius numbers are the Frobenius number and its half) cannot be pseudo-symmetric,

- the gluing of two nonsymmetric almost symmetric numerical semigroup is not almost symmetric ( $S$ is almost symmetric if the cardinality of $\mathbb{N} \backslash S$ equals $(\mathrm{F}(S)+\mathrm{t}(S)) / 2)$.

Let $S$ be an affine semigroup, and let $\mathbf{s} \in S \backslash\{0\}$. The Apéry set of $\mathbf{s}$ in $S$ is the set

$$
\operatorname{Ap}(S, \mathbf{s})=\{\mathbf{x} \in S \mid \mathbf{x}-\mathbf{s} \notin S\}
$$

This set has, in general, infinitely many elements. If $S$ is a numerical semigroup and $s \in S \backslash\{0\}$, then $\operatorname{Ap}(S, s)$ has exactly $s$ elements (one for each congruent class modulo $s$ ). Let $m$ be the least positive integer belonging to $S$, which is known as the multiplicity of $S$, and assume that $S$ is minimally generated by $\left\{n_{1}, \ldots, n_{k}\right\}$, with $n_{1}<\cdots<n_{k}$. 
Clearly, $n_{1}=m$ and $\operatorname{Ap}(S, m) \subseteq\left\{\sum_{i=2}^{k} a_{i} n_{i} \mid a_{i} \leq \alpha_{i}, i \in\{2, \ldots, k\}\right\}$, with $\alpha_{i}=\max \left\{k \in \mathbb{N} \mid k n_{i} \in \operatorname{Ap}(S, m)\right\}$. When the equality holds, we say that the Apéry set of $S$ is $\alpha$-rectangular. Theorem 2.3 in [10] shows that every numerical semigroup with $\alpha$-rectangular Apéry set other than $\mathbb{N}$ can be constructed by gluing a numerical semigroup with the same property and a copy of $\mathbb{N}$.

For a given affine semigroup $S$ and a field $K$, the semigroup ring $K[S]$ is defined as $K[S]=\bigoplus_{\mathbf{s} \in S} K t^{\mathbf{s}}$ with $t$ an indeterminate. Addition is performed componentwise, and the product is calculated by using distributive law and $t^{\mathbf{s}} t^{\mathbf{s}^{\prime}}=t^{\mathbf{s}+\mathbf{s}^{\prime}}$ for all $\mathbf{s}, \mathbf{s}^{\prime} \in S$. If $S$ is a numerical semigroup, then $K[S]$ is a subring of $K[t]$. Recently ([13]), the following property has been shown to be preserved under gluing of numerical semigroups: for every relative $I$ ideal of $K[S]$ generated by two monomials, $I \otimes_{K[S]} I^{-1}$ has nontrivial torsion. This partly solves a conjecture stated by Huneke and Wiegand (see [13] for details; also the restriction of being generated by just two elements can be removed if we take $S_{2}$ as a copy of $\mathbb{N}$ ).

If $S$ is a numerical semigroup minimally generated by $\left\{n_{1}, \ldots, n_{k}\right\}$, then $\mathfrak{m}=\left(t^{n_{1}}, \ldots, t^{n_{k}}\right)$ is the unique maximal ideal of the power series ring $R=K\left[\left[t^{n_{1}}, \ldots, t^{n_{k}}\right]\right]=K[[S]]$. The Hilbert function of the associated graded ring $\operatorname{gr}_{\mathfrak{m}}(R)=\bigoplus_{n \in \mathbb{N}} \mathfrak{m}^{n} / \mathfrak{m}^{n+1}$ is defined as $n \mapsto \operatorname{dim}_{K}\left(\mathfrak{m}^{n} / \mathfrak{m}^{n+1}\right)$. In [2], it is shown that, if the Hilbert functions of the associated graded rings of $K\left[\left[S_{1}\right]\right]$ and $K\left[\left[S_{2}\right]\right]$ are nondecreasing, then so is the Hilbert function of the associated graded ring of $K\left[\left[S_{1}+{ }_{d} S_{2}\right]\right]$ when the gluing is a "nice" gluing (see [2, Theorem 2.6] for details; this nice gluing has been also exploited in [16]).

Lastly, for $T=\left\langle a n_{1}, a n_{2}, a n_{3}, a n_{4}\right\rangle+{ }_{a b}\langle b\rangle$, Barucci and Fröberg have been able to compute the Betti numbers of the free resolution of $K[T]$ in terms of that of $K[S]$, with $S=\left\langle n_{1}, n_{2}, n_{3}, n_{4}\right\rangle([5])$.

2. Gluings and cones. Given an affine semigroup $S \subseteq \mathbb{N}^{m}$, denote by cone $(S)$ the cone spanned by $S$, that is,

$$
\operatorname{cone}(S)=\left\{q \mathbf{a} \mid q \in \mathbb{Q}_{\geq 0}, \mathbf{a} \in S\right\}
$$

Observe that cone $(S)$ is pointed (the only subspace included in it is $\{0\})$, because $S$ is reduced. 
Clearly, if $A$ is finite and generates $S$, then

$$
\mathrm{G}(S)=\left\{\sum_{\mathbf{a} \in A} z_{\mathbf{a}} \mathbf{a} \mid z_{\mathbf{a}} \in \mathbb{Z} \text { for all } \mathbf{a}\right\}
$$

and

$$
\operatorname{cone}(S)=\left\{\sum_{\mathbf{a} \in A} q_{\mathbf{a}} \mathbf{a} \mid q_{\mathbf{a}} \in \mathbb{Q}_{\geq 0} \text { for all } \mathbf{a}\right\}
$$

We will write $\operatorname{aff}(S)$ for the affine span of $S$, that is,

$$
\operatorname{aff}(S)=G(S) \otimes_{\mathbb{Z}} \mathbb{Q}
$$

As usual, we use the notation

$$
\langle A\rangle=\left\{\sum_{\mathbf{a} \in A} n_{\mathbf{a}} \mathbf{a} \mid n_{\mathbf{a}} \in \mathbb{N} \text { for all } \mathbf{a} \in A\right\}
$$

(all sums are finite, that is, if $A$ has infinitely many elements, all but a finite number of $z_{\mathbf{a}}, q_{\mathbf{a}}$ and $n_{\mathbf{a}}$ are zero).

Lemma 2.1. Let $\mathbf{r}_{1}, \ldots, \mathbf{r}_{k}, \mathbf{r}_{k+1}$ and $\mathbf{x} \in \operatorname{cone}\left(\mathbb{N}^{m}\right) \backslash\{0\}$, for some positive integers $m$ and $k$. If cone $\left(\mathbf{r}_{1}, \ldots, \mathbf{r}_{k}\right)=\operatorname{cone}\left(\mathbf{r}_{1}, \ldots, \mathbf{r}_{k}, \mathbf{r}_{k+1}\right)$, then the following conditions are equivalent:

(i) There exist $q_{1}, \ldots q_{k} \in \mathbb{Q}_{>0}$ such that $\mathbf{x}=q_{1} \mathbf{r}_{1}+\cdots+q_{k} \mathbf{r}_{k}$.

(ii) There exist $q_{1}^{\prime}, \ldots, q_{k+1}^{\prime} \in \mathbb{Q}_{>0}$ such that $\mathbf{x}=q_{1}^{\prime} \mathbf{r}_{1}+\cdots+q_{k}^{\prime} \mathbf{r}_{k}+$ $q_{k+1}^{\prime} \mathbf{r}_{k+1}$.

Proof. Observe that, from the hypothesis, $\mathbf{r}_{k+1} \in \operatorname{cone}\left(\mathbf{r}_{1}, \ldots, \mathbf{r}_{k}\right)$, and thus there exists $t_{1}, \ldots, t_{k} \in \mathbb{Q}_{\geq 0}$ such that $\mathbf{r}_{k+1}=t_{1} \mathbf{r}_{1}+\cdots+t_{k} \mathbf{r}_{k}$. From this, it easily follows that (ii) implies (i).

Assume that there exist $q_{1}, \ldots q_{k} \in \mathbb{Q}_{>0}$ such that $\mathbf{x}=q_{1} \mathbf{r}_{1}+\cdots+$ $q_{k} \mathbf{r}_{k}$. Let $N \in \mathbb{N}$ be such that, for all $i \in\{1, \ldots, k\}, t_{i} / N<q_{i}$ (this is possible since $q_{i}>0$ for all $i$ ). Take $q_{i}^{\prime}=q_{i}-t_{i} / N$ (which is a positive rational number) for all $i \in\{1, \ldots, k\}$, and $q_{k+1}^{\prime}=1 / N$. Then, $q_{1}^{\prime} \mathbf{r}_{1}+$ $\cdots+q_{k}^{\prime} \mathbf{r}_{k}+q_{k+1}^{\prime} \mathbf{r}_{k+1}=q_{1} \mathbf{r}_{1}+\cdots+q_{k} \mathbf{r}_{k}-1 / N \mathbf{r}_{k+1}+1 / N \mathbf{r}_{k+1}=\mathbf{x}$. 
Given $\mathbf{r}_{1}, \ldots, \mathbf{r}_{k} \in \operatorname{cone}\left(\mathbb{N}^{m}\right) \backslash\{0\}$, we define the relative interior of $\operatorname{cone}\left(\mathbf{r}_{1}, \ldots, \mathbf{r}_{k}\right)$ by

$$
\operatorname{relint}\left(\operatorname{cone}\left(\mathbf{r}_{1}, \ldots, \mathbf{r}_{k}\right)\right)=\left\{q_{1} \mathbf{r}_{1}+\cdots+q_{k} \mathbf{r}_{k} \mid q_{1}, \ldots, q_{k} \in \mathbb{Q}_{>0}\right\} .
$$

Observe that the relative interior of a cone, $C$, is the topological interior of $C$ in its affine span, aff $\left(\mathbf{r}_{1}, \ldots, \mathbf{r}_{k}\right)$, with the subspace topology.

For $A \subseteq \mathbb{N}^{m}$, we say that $F$ is a face of $\operatorname{cone}(A)$ if $F \neq \emptyset$ and there exists $\mathbf{c} \in \mathbb{Q}^{m} \backslash\{0\}$ such that

- $F=\{\mathbf{x} \in \operatorname{cone}(A) \mid \mathbf{c} \cdot \mathbf{x}=0\}$ and

- $\mathbf{c} \cdot \mathbf{y} \geq 0$ for all $\mathbf{y} \in \operatorname{cone}(A)$.

An element $\mathbf{a} \in A$ is an extremal ray of $\operatorname{cone}(A)$ if $\mathbb{Q}_{\geq 0}$ a is a onedimensional face of cone $(A)$.

Now, according to Lemma 2.1, if $A$ is the minimal system of generators of an affine semigroup $S \subseteq \mathbb{N}^{m}$, then we can say that $\mathbf{x} \in \operatorname{relint}(\operatorname{cone}(S))$ if and only if $\mathbf{x} \in \operatorname{relint}(\operatorname{cone}(A))$, even if $A$ contains elements that are not extremal rays. We get also the following consequence.

Proposition 2.2. Let $A$ be a nonempty subset of $\mathbb{N}^{m}$, with $m$ a positive integer. Assume that $A=A_{1} \cup A_{2}$ is a nontrivial partition of $A$. Then $\operatorname{relint}(\operatorname{cone}(A))=\operatorname{relint}\left(\operatorname{cone}\left(A_{1}\right)\right)+\operatorname{relint}\left(\operatorname{cone}\left(A_{2}\right)\right)$.

Proof. Obviously, if $\mathbf{x}_{i} \in \operatorname{relint}\left(\operatorname{cone}\left(A_{i}\right)\right), i \in\{1,2\}$, then $\mathbf{x}_{1}+\mathbf{x}_{2} \in$ relint $(\operatorname{cone}(A))$. Now, consider $\mathbf{x} \in \operatorname{relint}(\operatorname{cone}(A))$. Without loss of generality, we may assume that $\mathbf{x}=\sum_{\mathbf{a} \in A} q_{\mathbf{a}} \mathbf{a}$ with $q_{\mathbf{a}} \in \mathbb{Q}_{>0}$. Thus, by taking $\mathbf{x}_{i}=\sum_{\mathbf{a} \in A_{i}} q_{\mathbf{a}} \mathbf{a}$, we are done.

Notice that, if $S$ is the gluing of $S_{1}$ and $S_{2}$ by $\mathbf{d}$, then

$\mathbf{d} \notin \operatorname{relint}(\operatorname{cone}(S))$ implies $\mathbf{d} \notin \operatorname{relint}\left(\operatorname{cone}\left(S_{1}\right)\right) \cap \operatorname{relint}\left(\operatorname{cone}\left(S_{2}\right)\right)$.

Otherwise, we may take $\mathbf{x}_{i}=(1 / 2) \mathbf{d}, i \in\{1,2\}$.

Proposition 2.3. Let $A$ be a nonempty subset of $\mathbb{N}^{m}$, with $m$ a positive integer. Assume that $A=A_{1} \cup A_{2}$ is a nontrivial partition of $A$. Let $F$ be a face of cone $(A)$. Then every $\mathbf{x} \in F$ can be expressed as $\mathbf{x}_{1}+\mathbf{x}_{2}$ with $\mathbf{x}_{i}$ in a face of cone $\left(A_{i}\right), i \in\{1,2\}$. 
Proof. Let $\mathbf{x} \in F$. Then there exists $\mathbf{c} \in \mathbb{Q}^{m} \backslash\{0\}$ such that $\mathbf{c} \cdot \mathbf{x}=0$ and $\mathbf{c} \cdot \mathbf{y} \geq 0$ for all $\mathbf{y} \in \operatorname{cone}(A)$. Notice that $\operatorname{cone}(A)=$ $\operatorname{cone}\left(A_{1}\right)+\operatorname{cone}\left(A_{2}\right)$. Hence, there exists $\mathbf{x}_{i} \in \operatorname{cone}\left(A_{i}\right), i \in\{1,2\}$, such that $\mathbf{x}=\mathbf{x}_{1}+\mathbf{x}_{2}$. As cone $\left(A_{i}\right) \subseteq \operatorname{cone}(A), \mathbf{c} \cdot \mathbf{y}_{i} \geq 0$, for $i \in\{1,2\}$ and all $\mathbf{y}_{i} \in \operatorname{cone}\left(A_{i}\right)$. Hence, $0=\mathbf{c} \cdot \mathbf{x}=\mathbf{c} \cdot \mathbf{x}_{1}+\mathbf{c} \cdot \mathbf{x}_{2}$ forces $\mathbf{c} \cdot \mathbf{x}_{1}=\mathbf{c} \cdot \mathbf{x}_{2}=0$. We conclude that $\mathbf{x}_{i}$ is in the face $\left\{\mathbf{x} \in \mathbf{Q}^{n} \mid \mathbf{c} \cdot \mathbf{x}=0\right\} \cap \operatorname{cone}\left(A_{i}\right)$ of $\operatorname{cone}\left(A_{i}\right), i \in\{1,2\}$.

We end this section by giving an affine-geometric characterization of gluings (Corollary 2.5). First we show how the cones in a gluing intersect.

Proposition 2.4. Let $S$ be an affine semigroup and $\mathbf{d} \in \mathbb{N}^{n} \backslash\{0\}$. If $S=S_{1}+{ }_{\mathrm{d}} S_{2}$, then

$$
\operatorname{cone}\left(S_{1}\right) \cap \operatorname{cone}\left(S_{2}\right)=\mathbf{d} \mathbb{Q} \geq 0 .
$$

Proof. By definition, $\mathbf{d} \in S_{1} \cap S_{2}$ and, clearly, $\mathbf{d} \mathbb{Q}_{\geq 0} \subseteq \operatorname{cone}\left(S_{1}\right) \cap$ $\operatorname{cone}\left(S_{2}\right)$. If $\mathbf{d}^{\prime} \in \operatorname{cone}\left(S_{1}\right) \cap \operatorname{cone}\left(S_{2}\right)$, then $\mathbf{d}^{\prime}=\left(z_{1} / t_{1}\right) \mathbf{a}_{1}=\left(z_{2} / t_{2}\right) \mathbf{a}_{2}$, with $z_{1}, z_{2}, t_{1}, t_{2} \in \mathbb{N}$, and $\mathbf{a}_{i} \in S_{i}, i \in\{1,2\}$. Hence, $t_{1}, t_{2} \mathbf{d}^{\prime} \in$ $\mathrm{G}\left(S_{1}\right) \cap \mathrm{G}\left(S_{2}\right)=\mathbf{d} \mathbb{Z}$, that is, $\mathbf{d}^{\prime} \in \mathbf{d} \mathbb{Q}_{\geq 0}$.

The above result may also be obtained as a consequence of $[\mathbf{1 8}$, Lemma 4.2].

Observe that the converse statement is not true, as the following simple example shows. Let $S$ be semigroup generated by the columns of the matrix

$$
A=\left(\begin{array}{lll|lll}
4 & 3 & 2 & 3 & 1 & 0 \\
0 & 1 & 2 & 3 & 3 & 4
\end{array}\right),
$$

and let $S_{1}$ and $S_{2}$ be the semigroups generated by the three first and the three last columns of $A$, respectively. In this case, $\mathbf{d}:=(6,6)^{\top} \in S_{1} \cap S_{2}$ and cone $\left(S_{1}\right) \cap \operatorname{cone}\left(S_{2}\right)=\mathbf{d} \mathbb{Q}_{\geq 0}$. However, $S_{1}$ and $S_{2}$ cannot be glued by $\mathbf{d}$ because $\mathrm{G}\left(S_{1}\right) \cap \mathrm{G}\left(S_{2}\right)$ has rank 2 ; indeed, $3(2,2)=2(3,3)$ and $(0,4)=-2(4,0)+2(3,1)+(2,2)$.

Corollary 2.5. Let $S$ be an affine semigroup minimally generated by A. Let $A=A_{1} \cup A_{2}$ be a nontrivial partition of $A$, and let $S_{i}=\left\langle A_{i}\right\rangle$, $i \in\{1,2\}$. Set $V=\operatorname{aff}\left(S_{1}\right) \cap \operatorname{aff}\left(S_{2}\right)$. Then, $S=S_{1}+_{\mathbf{d}} S_{2}$ for some 
$\mathbf{d} \in \mathbb{N}^{n} \backslash\{0\}$, if and only if $V=\mathbf{d} \mathbb{Q}$ and $S \cap V=\left(S_{1} \cap V\right)+_{\mathbf{d}}\left(S_{2} \cap V\right)$ for some $\mathbf{d} \in \mathbb{N}^{n} \backslash\{0\}$.

Proof. If $S=S_{1}+_{\mathbf{d}} S_{2}$ for some $\mathbf{d} \in \mathbb{N}^{n} \backslash\{0\}$, by an argument similar to that given in the proof of Proposition 2.4, we have that $V=\mathbf{d} \mathbb{Q}$. Now, since $\mathbf{d} \in\left(S_{1} \cap V\right) \cap\left(S_{2} \cap V\right)$ and $\mathrm{G}\left(S_{1} \cap V\right) \cap \mathrm{G}\left(S_{2} \cap V\right)=$ $\mathrm{G}\left(S_{1}\right) \cap \mathrm{G}\left(S_{2}\right)=\mathbf{d} \mathbb{Z}$, we conclude that $S \cap V$ is the gluing of $S_{1} \cap V$ and $S_{2} \cap V$ by d. Conversely, let $V=\mathbf{d} \mathbb{Q}$. Since $\mathrm{G}\left(S_{1}\right) \cap \mathrm{G}\left(S_{2}\right)=$ $\mathrm{G}\left(S_{1} \cap V\right) \cap \mathrm{G}\left(S_{2} \cap V\right)=\mathbf{d} \mathbb{Z}$ and $\mathbf{d} \in\left(S_{1} \cap V\right) \cap\left(S_{2} \cap V\right)=S_{1} \cap S_{2}$, because $\mathrm{G}\left(S_{1}\right) \cap \mathrm{G}\left(S_{2}\right) \subset V$, we are done.

Let $S$ be the semigroup generated by the columns of the following matrix

$$
A=\left(\begin{array}{lll|lll}
4 & 3 & 2 & 3 & 3 & 3 \\
0 & 1 & 2 & 3 & 2 & 0 \\
0 & 0 & 0 & 0 & 1 & 3
\end{array}\right),
$$

and let $S_{1}\left(S_{2}\right.$, respectively) be the semigroup generated by the three first (last, respectively) columns of $A$. Clearly, $V=\operatorname{aff}\left(S_{1}\right) \cap \operatorname{aff}\left(S_{2}\right)=$ $(1,1,0)^{\top} \mathbb{Q}$. Now, since $S_{1} \cap V \cong 2 \mathbb{N}, S_{2} \cap V \cong 3 \mathbb{N}$ and $S \cap V \cong 2 \mathbb{N}+{ }_{6} 3 \mathbb{N}$, in the light of the above corollary, we conclude that $S=S_{1}+{ }_{\mathbf{d}} S_{2}$, with $\mathbf{d}=(6,6,0)^{\top}$.

3. Gluings and Frobenius vectors. Let $S$ be an affine semigroup. We say that $S$ has a Frobenius vector if there exists $\mathbf{f} \in \mathrm{G}(S) \backslash S$ such that

$$
\mathbf{f}+\operatorname{relint}(\operatorname{cone}(S)) \cap \mathrm{G}(S) \subseteq S \backslash\{0\} \subseteq S .
$$

Notice that $\mathbf{f}+(\operatorname{relint}(\operatorname{cone}(S)) \cap \mathrm{G}(S)) \subseteq S \backslash\{0\}$ is equivalent to $(\mathbf{f}+\operatorname{relint}(\operatorname{cone}(S))) \cap \mathrm{G}(S) \subseteq S \backslash\{0\}$, and thus we omit the parentheses in the above condition.

We are going to prove that, if $S_{1}$ and $S_{2}$ have Frobenius vectors, then so does $S=S_{1}+_{\mathrm{d}} S_{2}$.

Theorem 3.1. Let $S$ be an affine semigroup. Assume that $S=$ $S_{1}+_{\mathbf{d}} S_{2}$. If $S_{1}$ and $S_{2}$ have Frobenius vectors, so does $S$. Moreover, if $\mathbf{f}_{1}$ and $\mathbf{f}_{2}$ are, respectively, Frobenius vectors of $S_{1}$ and $S_{2}$, then

$$
\mathbf{f}=\mathbf{f}_{1}+\mathbf{f}_{2}+\mathbf{d}
$$

is a Frobenius vector of $S$. 
Proof. Let $G_{1}=\mathrm{G}\left(S_{1}\right), G_{2}=\mathrm{G}\left(S_{2}\right)$ and $G=\mathrm{G}(S)$. Clearly $G=G_{1}+G_{2}$, since $S=S_{1}+S_{2}$.

We start by proving that $\mathbf{f} \in G \backslash S$. As $\mathbf{f}_{1} \in G_{1}, \mathbf{f}_{2} \in G_{2}$ and $\mathbf{d} \in G_{1} \cap G_{2}$, we have $\mathbf{f} \in G$. Assume that $\mathbf{f} \in S$. Then there exist $\mathbf{s}_{1} \in S_{1}$ and $\mathbf{s}_{2} \in S_{2}$ such that $\mathbf{f}=\mathbf{s}_{1}+\mathbf{s}_{2}$. Then $\mathbf{f}_{1}+\mathbf{d}-\mathbf{s}_{1}=\mathbf{s}_{2}-\mathbf{f}_{2} \in G_{1} \cap G_{2}=\mathbf{d} \mathbb{Z}$. So, we can find $k \in \mathbb{Z}$ such that $\mathbf{f}_{1}+\mathbf{d}-\mathbf{s}_{1}=\mathbf{s}_{2}-\mathbf{f}_{2}=k \mathbf{d}$. If $k \leq 0$, then $\mathbf{f}_{2}=\mathbf{s}_{2}-k \mathbf{d} \in S_{2}$, a contradiction. If $k>0$, then $\mathbf{f}_{1}=\mathbf{s}_{1}+(k-1) \mathbf{d} \in S_{1}$, which is also impossible, and this proves that $\mathbf{f} \notin S$.

In order to simplify the notation, set $C_{1}=\operatorname{relint}\left(\operatorname{cone}\left(S_{1}\right)\right), C_{2}=$ $\operatorname{relint}\left(\operatorname{cone}\left(S_{2}\right)\right)$ and $C=\operatorname{relint}(\operatorname{cone}(S))$. Now, let us prove that, for all $\mathbf{x} \in C \cap G$, we have that $\mathbf{f}+\mathbf{x} \in S$. Since $\mathbf{f}+\mathbf{x} \in G$, there must be $\mathbf{g}_{1} \in G_{1}$ and $\mathbf{g}_{2} \in G_{2}$ such that $\mathbf{f}+\mathbf{x}=\mathbf{g}_{1}+\mathbf{g}_{2}$. In light of Proposition 2.2, there exists $\mathbf{x}_{1} \in C_{1}$ and $\mathbf{x}_{2} \in C_{2}$ such that $\mathbf{x}=\mathbf{x}_{1}+\mathbf{x}_{2}$. Then $\mathbf{f}+\mathbf{x}=\mathbf{f}_{1}+\mathbf{f}_{2}+\mathbf{d}+\mathbf{x}_{1}+\mathbf{x}_{2}=\mathbf{g}_{1}+\mathbf{g}_{2}$. Let $t \in \mathbb{Z}_{>0}$ be such that $\mathbf{s}_{1}=t \mathbf{x}_{1} \in S_{1}$ and $\mathbf{s}_{2}=t \mathbf{x}_{2} \in S_{2}$. This yields $t \mathbf{f}_{1}+t \mathbf{d}+\mathbf{s}_{1}-t \mathbf{g}_{1}=t \mathbf{g}_{2}-t \mathbf{f}_{2}-\mathbf{s}_{2}=k \mathbf{d}$ for some integer $k$. Assume that $k \leq 0$. Then $t \mathbf{f}_{1}+\mathbf{s}_{1}+(t-k) \mathbf{d}=t \mathbf{g}_{1}$, and thus $\mathbf{f}_{1}+\left(\mathbf{x}_{1}+(t-k) / t \mathbf{d}\right)=\mathbf{g}_{1}$. Observe that $\mathbf{x}_{1}+(t-k) / t \mathbf{d} \in C_{1}$, which implies that $\mathbf{g}_{1} \in S_{1}$ because $\mathbf{f}_{1}$ is a Frobenius vector for $S_{1}$.

Let $n$ be the maximum nonnegative integer such that $\mathbf{g}_{1}-n d \in S_{1}$. Hence, $\mathbf{g}_{1}-(n+1) \mathbf{d}=\mathbf{f}_{1}+\mathbf{x}_{1}+(t-k / t) \mathbf{d}-(n+1) \mathbf{d} \notin S_{1}$, and consequently $t n+k>0$, since otherwise $(t-k / t)-(n+1) \geq 0$, which leads to $\mathbf{x}_{1}+(t-k / t) \mathbf{d}-(n+1) \mathbf{d} \in C_{1}$, yielding $\mathbf{g}_{1}-(n+1) \mathbf{d} \in S_{1}$, a contradiction. Now, $t \mathbf{g}_{2}-t \mathbf{f}_{2}-\mathbf{s}_{2}+t n \mathbf{d}=(t n+k) \mathbf{d}$, which means that $\mathbf{g}_{2}+n \mathbf{d}=\mathbf{f}_{2}+\mathbf{x}_{2}+(t n+k / t) \mathbf{d}$. As $\mathbf{x}_{2}+(t n+k / t) \mathbf{d} \in C_{2}$ and $\mathbf{f}_{2}$ is a Frobenius vector for $S_{2}$, we deduce that $\mathbf{g}_{2}+n \mathbf{d} \in S_{2}$. Finally, $\mathbf{f}+\mathbf{x}=\mathbf{g}_{1}+\mathbf{g}_{2}=\left(\mathbf{g}_{1}-n \mathbf{d}\right)+\left(\mathbf{g}_{2}+n \mathbf{d}\right) \in S_{1}+S_{2}=S$.

If $k \geq 0$, then $t \mathbf{f}_{2}+\mathbf{s}_{2}+t \mathbf{d}-t \mathbf{g}_{2}=t \mathbf{g}_{1}-t \mathbf{f}_{2}-\mathbf{s}_{1}=-k \mathbf{d}$, and we repeat the above argument by swapping $\mathbf{g}_{1}$ and $\mathbf{g}_{2}$.

If $A$ is a set of positive integers, and $S=\langle A\rangle$, then $T=S / \operatorname{gcd}(A)$ is a numerical semigroup, and $\mathrm{F}(T)=\max (\mathbb{N} \backslash T)$. It follows easily that $\mathrm{F}(S)=\operatorname{gcd}(A) \mathrm{F}(T)$. Recall that the conductor of $T$ is defined as the Frobenius number of $T$ plus one. Hence, Theorem 3.1 generalizes the well-known formula for the gluing of two submonoids of $\mathbb{N}$ ([11, Proposition 10 (i)]). 
Lemma 3.2. Let $S$ be an affine semigroup minimally generated by $A$. If $A$ is a set of linearly independent elements, then $\mathbf{f}=-\sum_{\mathbf{a} \in A} \mathbf{a}$ is a Frobenius vector for $S$.

Proof. Let $\mathbf{x} \in \operatorname{relint}(\operatorname{cone}(S)) \cap G(S)$. Then $\mathbf{x}=\sum_{\mathbf{a} \in A} q_{\mathbf{a}} \mathbf{a}=$ $\sum_{\mathbf{a} \in A} z_{\mathbf{a}} \mathbf{a}$, with $q_{\mathbf{a}} \in \mathbb{Q}_{>0}$ and $z_{\mathbf{a}} \in \mathbb{Z}$ for all a. Since the elements in $A$ are linearly independent, this forces $z_{\mathbf{a}}=q_{\mathbf{a}}$ for all $\mathbf{a}$; in particular, $z_{\mathbf{a}}-1 \geq 0$ for all $\mathbf{a}$. Hence $\mathbf{f}+\mathbf{x}=\sum_{\mathbf{a} \in A}\left(z_{\mathbf{a}}-1\right) \mathbf{a} \in S$.

Since every complete intersection affine semigroup has either no relations (free in the categorical sense, that is, its minimal set of generators is a set of linearly independent vectors) or it is the gluing of two affine semigroups ([12]), we get the following result.

Theorem 3.3. Let $S$ be a complete intersection affine semigroup. Then $S$ has a Frobenius vector.

Remark 3.4. Let $S=S_{1}+_{\mathrm{d}} S_{2}$ be the gluing of $S_{1}$ and $S_{2}$ by $\mathbf{d}$, and assume that $S_{2}=\langle\mathbf{v}\rangle$. Hence, $\mathbf{d}=\theta \mathbf{v}$ for some $\theta \in \mathbb{N}$. Clearly $-\mathbf{v}$ is a Frobenius vector for $S_{2}$ (Lemma 3.2), and, if $S_{1}$ has a Frobenius vector $\mathbf{f}_{1}$, then the formula of Theorem 3.1 implies that $\mathbf{f}=\mathbf{f}_{1}-\mathbf{v}+\theta \mathbf{v}=\mathbf{f}_{1}+(\theta-1) \mathbf{v}$ is a Frobenius vector of $S$. More generally let $\mathbf{v}_{1}, \ldots, \mathbf{v}_{e}$ be a set of $\mathbb{Q}$ linearly independent vectors of $\mathbb{N}^{e}$. Let $S_{0}=\left\langle\mathbf{v}_{1}, \ldots, \mathbf{v}_{e}\right\rangle$, and let $\mathbf{v}_{e+1}, \ldots, \mathbf{v}_{e+h}$ be a set of vectors of $\mathbb{N}^{e} \cap \operatorname{cone}\left(\mathbf{v}_{1}, \ldots, \mathbf{v}_{e}\right)$. Set $S_{i}=\left\langle\mathbf{v}_{1}, \ldots, \mathbf{v}_{e+i}\right\rangle$ for all $1 \leq i \leq h$, and assume that $S_{i}=S_{i-1}+{ }_{\theta_{i} \mathbf{v}_{i}}\left\langle\mathbf{v}_{i}\right\rangle$ (such semigroups are called free semigroups). A Frobenius vector $\mathbf{f}_{0}$ of $S_{0}$ being $\mathbf{f}_{0}=-\sum_{k=1}^{e} \mathbf{v}_{k}$ (Lemma 3.2), it follows that

$$
\mathbf{f}_{i}=\sum_{j=1}^{i}\left(\theta_{j}-1\right) \mathbf{v}_{j}-\sum_{k=1}^{e} \mathbf{v}_{k}
$$

is a Frobenius vector of $S_{i}$. This formula has also been proved by the first author in [3], who gave the following uniqueness condition: this Frobenius vector $\mathbf{f}$ is minimal with respect to the order induced by cone $(S)$, that is, for every other Frobenius vector $\mathbf{f}^{\prime}$ of $S, \mathbf{f}^{\prime} \in$ f $+\operatorname{cone}(S)$.

We recall that a reduced affine semigroup $S$ is said to be simplicial if there are linearly independent elements $\mathbf{a}_{1}, \ldots, \mathbf{a}_{n} \in S$ such that 
$\operatorname{cone}(S)=\operatorname{cone}\left(\mathbf{a}_{1}, \ldots, \mathbf{a}_{n}\right)$. Under this hypothesis, conditions for the existence and conditions for uniqueness of a Frobenius vector of $S$ are given in [1].

Formula (3.1) is a special case of the following general formula for a Frobenius vector of a complete intersection affine semigroup.

Remark 3.5. Recall that, according to [12], any complete intersection affine semigroup is either generated by a set of linearly independent vectors or it is a gluing of two complete intersection numerical semigroups. Thus, repeating this argument recursively, if $S$ is a complete intersection affine semigroup $A$, then there exists a partition $A_{1} \cup \cdots \cup A_{t}=A$ such that $A_{i}$ are sets of linearly independent vectors and

$$
S=S_{1}+_{\mathbf{d}_{1}} S_{2}+{ }_{\mathbf{d}_{2}} \cdots+{ }_{\mathbf{d}_{t-1}} S_{t},
$$

with $S_{i}=\left\langle A_{i}\right\rangle$. From Theorem 3.1 and Lemma 3.2, it follows that

$$
\sum_{i=1}^{t-1} \mathbf{d}_{i}-\sum_{\mathbf{a} \in A} \mathbf{a}
$$

is a Frobenius vector for $S$.

Next, we show that this Frobenius vector is unique in the sense defined above.

Proposition 3.6. Let $S$ be a complete intersection affine semigroup, and let $\mathbf{f}$ be defined as in (3.2). Then, for every face $F$ of cone $(S)$, $(\mathbf{f}+F) \cap S$ is empty.

Proof. Since either $S$ is free or the gluing of two complete intersection affine semigroups $S_{1}$ and $S_{2}$, we proceed by induction. If $S$ is free, then Lemma 3.2 asserts that $\mathbf{f}=-\sum_{\mathbf{a} \in A}$ a, with $A$ the minimal generating set of $S$. Clearly, in this case, the assertion is true.

Now assume that $S=S_{1}+\mathbf{d} S_{2}$ for some $\mathbf{d} \in S_{1} \cap S_{2}$. From Theorem 3.1, $\mathbf{f}=\mathbf{f}_{1}+\mathbf{f}_{2}+\mathbf{d}$, where $\mathbf{f}_{i}, i \in\{1,2\}$, is also defined by (3.2). By the induction hypothesis, for every face $F_{i}$ of cone $\left(S_{i}\right)$, $i \in\{1,2\},\left(\mathbf{f}_{i}+F_{i}\right) \cap S_{i}=\emptyset$. 
Assume, to the contrary, that there exists $\mathbf{x} \in F$ such that $\mathbf{f}_{1}+$ $\mathbf{f}_{2}+\mathbf{d}+\mathbf{x} \in S$. According to Proposition 2.3, there exists $\mathbf{x}_{i} \in F_{i}$, $i \in\{1,2\}$, such that $\mathbf{x}=\mathbf{x}_{1}+\mathbf{x}_{2}$, for some face $F_{i}$ of cone $\left(S_{i}\right)$. Hence, there are $\mathbf{s}_{1} \in S_{1}$ and $\mathbf{s}_{2} \in S_{2}$ such that $\mathbf{f}_{1}+\mathbf{f}_{2}+\mathbf{d}+\mathbf{x}_{1}+\mathbf{x}_{2}=\mathbf{s}_{1}+\mathbf{s}_{2}$. Then $\mathbf{f}_{1}+\mathbf{x}_{1}-\mathbf{s}_{1}=\mathbf{s}_{2}-\mathbf{f}_{2}-\mathbf{d}-\mathbf{x}_{2}=k \mathbf{d}$ for some integer $k$. As by the induction hypothesis $\mathbf{f}_{1}+\mathbf{x}_{1} \notin S_{1}$, we deduce $k<0$. Therefore, $\mathbf{f}_{2}+\mathbf{x}_{2}=\mathbf{s}_{2}-(k+1) \mathbf{d}$. But $\mathbf{f}_{2}+\mathbf{x}_{2} \notin S_{2}$, which forces $k+1>0$, or equivalently, $k \geq 0$. But this is in contradiction with $k<0$.

Theorem 3.7. Let $S$ be a complete intersection, and let $\mathbf{f}$ be as in (3.2). Assume that $\mathbf{f}^{\prime}$ is another Frobenius vector of $S$. Then $\mathbf{f}^{\prime} \in \mathbf{f}+\operatorname{cone}(S)$.

Proof. Write $\mathbf{f}=\mathbf{a}-\mathbf{b}$ and $\mathbf{f}^{\prime}=\mathbf{a}^{\prime}-\mathbf{b}^{\prime}$ with $\mathbf{a}, \mathbf{a}^{\prime}, \mathbf{b}, \mathbf{b}^{\prime} \in S$, and let $\mathbf{c} \in \operatorname{relint}(\operatorname{cone}(S))$. Then $\mathbf{x}=\mathbf{f}+\mathbf{b}+\mathbf{a}^{\prime}+\mathbf{c}=\mathbf{f}^{\prime}+\mathbf{b}^{\prime}+\mathbf{a}+\mathbf{c} \in$ $(\mathbf{f}+\operatorname{relint}(\operatorname{cone}(S))) \cap\left(\mathbf{f}^{\prime}+\operatorname{relint}(\operatorname{cone}(S))\right)$.

Assume that $\mathbf{f}^{\prime} \notin \mathbf{f}+\operatorname{cone}(S)$. Then the segment joining $\mathbf{f}^{\prime}$ and $\mathbf{x}$ cuts some face of $\mathbf{f}+\operatorname{cone}(S)$. Denote by $\mathbf{f}+F$ this face, and let $\mathbf{f}+\mathbf{y}$ be this intersection point $(\mathbf{y} \in F$ and $F$ is a face of cone $(S))$. There exists a positive integer $k$ such that $k \mathbf{y}$ is in $S$, and thus $\mathbf{f}+k \mathbf{y} \in \mathrm{G}(S) \cap(\mathbf{f}+F)$. Notice that $\mathbf{f}+\mathbf{y}=\mathbf{f}^{\prime}+\mathbf{y}^{\prime}$ for some $\mathbf{y}^{\prime} \in \operatorname{relint}(\operatorname{cone}(S))$. As $\mathbf{y} \in F,(k-1) \mathbf{y} \in \operatorname{cone}(S)$, and consequently $\mathbf{f}+k \mathbf{y}=\mathbf{f}^{\prime}+\left(\mathbf{y}^{\prime}+(k-1) \mathbf{y}\right) \in \mathbf{f}^{\prime}+\operatorname{relint}(\operatorname{cone}(S))$. Hence, $\mathbf{f}+k \mathbf{y} \in\left(\mathbf{f}^{\prime}+\operatorname{relint}(\operatorname{cone}(S))\right) \cap \mathrm{G}(S) \subseteq S$, in contradiction with Proposition 3.6.

4. Gluings and Hilbert series. The Hilbert series of $S$ is the Hilbert series associated to $K[S]: \mathrm{H}(S, \mathbf{x})=\sum_{\mathbf{s} \in S} \mathbf{x}^{\mathbf{s}}$, where for $\mathbf{s}=\left(s_{1}, \ldots, s_{m}\right) \in \mathbb{N}^{m}, \mathbf{x}^{\mathbf{s}}=x_{1}^{s_{1}} \cdots x_{m}^{s_{m}}$. This map is sometimes known in the literature as a generating function of $S$, and it has been shown to be of the form $g(S, \mathbf{x}) / \prod_{\mathbf{a} \in A}\left(1-\mathbf{x}^{\mathbf{a}}\right)$, with $A$ the minimal generating set of $S$ (see [6, subsection 7.3]).

The next lemma is a straightforward generalization of (4) in [21].

Lemma 4.1. Let $S$ be an affine semigroup, and let $\mathbf{m} \in S \backslash\{0\}$. Then

$$
\mathrm{H}(S, x)=\frac{1}{1-x^{\mathbf{m}}} \sum_{\mathbf{w} \in \operatorname{Ap}(S, \mathbf{m})} x^{\mathbf{w}} .
$$


Proof. It follows directly from the definition of $\operatorname{Ap}(S, \mathbf{m})$, that for every $\mathbf{s} \in S$, there exist unique $k \in \mathbb{N}$ and $\mathbf{w} \in \operatorname{Ap}(S, \mathbf{m})$ such that $\mathbf{s}=k \mathbf{m}+\mathbf{w}$. Hence,

$$
\mathrm{H}(S, \mathbf{x})=\sum_{\substack{k \in \mathbb{N} \\ \mathbf{w} \in \operatorname{Ap}(S, \mathbf{m})}} \mathbf{x}^{k \mathbf{m}+\mathbf{w}}=\sum_{k \in \mathbb{N}}\left(\mathbf{x}^{\mathbf{m}}\right)^{\mathbf{k}} \sum_{\mathbf{w} \in \operatorname{Ap}(S, \mathbf{m})} x^{\mathbf{w}}
$$

The proof follows by taking into account that $\sum_{k \in \mathbb{N}}\left(\mathbf{x}^{\mathbf{m}}\right)^{\mathbf{k}}=1 /(1-$ $\mathbf{x}^{\mathbf{m}}$ ).

The following result can also be understood as a generalization of (4) in [21], since for simplicial affine semigroups that are Cohen-Macaulay, the set $\bigcap_{i=1}^{m} \operatorname{Ap}\left(S, \mathbf{v}_{i}\right)$, with $\mathbf{v}_{1}, \ldots, \mathbf{v}_{m}$ a set of extremal rays of $S$, plays a similar role to the Apéry set of an element in a numerical semigroup (compare [24, Theorem 1.5] and [26, Lemma 2.6]).

Proposition 4.2. Let $S$ be a simplicial affine semigroup with extremal rays $\mathbf{v}_{1}, \ldots, \mathbf{v}_{m}$. Then $\mathrm{H}(S, \mathbf{x})=P(\mathbf{x}) / \prod_{i=1}^{m}\left(1-\mathbf{x}^{\mathbf{v}_{i}}\right)$, with $P(\mathbf{x})$ a polynomial.

Proof. Let $A p=\bigcap_{i=1}^{m} \operatorname{Ap}\left(S, \mathbf{v}_{i}\right)$. In view of [24, Section 1], this set is finite. Moreover, from [24, Theorem 1.5], we know that every element $\mathbf{s}$ in $S$ can be expressed uniquely as $\mathbf{s}=\sum_{i=1}^{m} a_{i} \mathbf{v}_{i}+\mathbf{w}$ with $a_{1}, \ldots, a_{d} \in \mathbb{N}$ and $\mathbf{w} \in A p$. Arguing as in Lemma 4.1,

$$
\mathrm{H}(S, \mathbf{x})=\sum_{\mathbf{s} \in S} \mathbf{x}^{\mathbf{s}}=\frac{\sum_{\mathbf{w} \in A p} \mathbf{x}^{\mathbf{w}}}{\prod_{i=1}^{m}\left(1-x^{\mathbf{v}_{i}}\right)},
$$

which concludes the proof.

Theorem 4.3. Let $S, S_{1}$ and $S_{2}$ be affine semigroups, and let $\mathbf{d} \in S$. Assume that $S=S_{1}+{ }_{\mathbf{d}} S_{2}$. Then

$$
\mathrm{H}\left(S_{1}+_{\mathbf{d}} S_{2}, \mathbf{x}\right)=\left(1-\mathbf{x}^{d}\right) \mathrm{H}\left(S_{1}, \mathbf{x}\right) \mathrm{H}\left(S_{2}, \mathbf{x}\right) .
$$

Proof. From (4.1),

$$
\mathrm{H}(S, \mathbf{x})=\frac{1}{1-\mathbf{x}^{\mathbf{d}}} \sum_{\mathbf{w} \in \operatorname{Ap}(S, \mathbf{d})} \mathbf{x}^{\mathbf{w}}
$$


From [22, Theorem 1.4], the mapping

$$
\operatorname{Ap}\left(S_{1}, \mathbf{d}\right) \times \operatorname{Ap}\left(S_{2}, \mathbf{d}\right) \longrightarrow \operatorname{Ap}(S, \mathbf{d}), \quad(x, y) \mapsto x+y,
$$

is a bijection, and thus, $\operatorname{Ap}(S, \mathbf{d})=\operatorname{Ap}\left(S_{1}, \mathbf{d}\right)+\operatorname{Ap}\left(S_{2}, \mathbf{d}\right)$. Hence,

$$
\begin{aligned}
\sum_{\mathbf{w} \in \operatorname{Ap}(S, \mathbf{d})} x^{\mathbf{w}} & =\sum_{\mathbf{w}_{1} \in \operatorname{Ap}\left(S_{1}, \mathbf{d}\right)} \sum_{\mathbf{w}_{2} \in \operatorname{Ap}\left(S_{2}, \mathbf{d}\right)} \mathbf{x}^{\mathbf{w}_{1}+\mathbf{w}_{2}} \\
& =\left(\sum_{\mathbf{w}_{1} \in \operatorname{Ap}\left(S_{1}, \mathbf{d}\right)} \mathbf{x}^{\mathbf{w}_{1}}\right)\left(\sum_{\mathbf{w}_{2} \in \operatorname{Ap}\left(S_{2}, \mathbf{d}\right)} \mathbf{x}^{\mathbf{w}_{2}}\right) .
\end{aligned}
$$

As

$$
\begin{aligned}
& \mathrm{H}\left(S_{1}, \mathbf{x}\right)=\frac{1}{1-\mathbf{x}^{\mathbf{d}}} \sum_{\mathbf{w}_{1} \in \operatorname{Ap}\left(S_{1}, \mathbf{d}\right)} x^{\mathbf{w}_{1}}, \\
& \mathrm{H}\left(S_{2}, \mathbf{x}\right)=\frac{1}{1-\mathbf{x}^{\mathbf{d}}} \sum_{\mathbf{w}_{2} \in \operatorname{Ap}\left(S_{2}, \mathbf{d}\right)} \mathbf{x}^{\mathbf{w}_{2}},
\end{aligned}
$$

we get

$$
\mathrm{H}(S, \mathbf{x})=\left(1-\mathbf{x}^{\mathbf{d}}\right) \mathrm{H}\left(S_{1}, \mathbf{x}\right) \mathrm{H}\left(S_{2}, \mathbf{x}\right) .
$$

If $S$ is a numerical semigroup $(\operatorname{gcd}(S)=1)$, and it is a gluing of $M_{1}$ and $M_{2}$, then $S_{1}=M_{1} / d_{1}$ and $S_{2}=M_{2} / d_{2}$ are also numerical semigroups, with $d_{i}=\operatorname{gcd}\left(M_{i}\right), i \in\{1,2\}$. Hence, $S=d_{1} S_{1}+d_{d_{1} d_{2}} d_{2} S_{2}$ and $\operatorname{lcm}\left(d_{1}, d_{2}\right)=d_{1} d_{2}$. We say in this setting that $S$ is a gluing of $S_{1}$ and $S_{2}$ at $d_{1} d_{2}$.

From the definition of the Hilbert series associated to a submonoid $M$ of $N$, it follows easily that, if $k \mid \operatorname{gcd}(M)$, then

$$
\mathrm{H}\left(M / k, x^{k}\right)=\mathrm{H}(M, x) .
$$

We get the following corollary.

Corollary 4.4. Let $S$ be a numerical semigroup. Assume that $S=$ $d_{1} S_{1}+{ }_{d_{1} d_{2}} d_{2} S_{2}$ is a gluing of the numerical semigroups $S_{1}$ and $S_{2}$. Then

$$
\mathrm{H}(S, x)=\left(1-x^{d_{1} d_{2}}\right) \mathrm{H}\left(S_{1}, x^{d_{1}}\right) \mathrm{H}\left(S_{2}, x^{d_{2}}\right) .
$$


Example 4.5. Let $S=\langle a, b\rangle$ with $a$ and $b$ coprime positive integers. Then $S=a \mathbb{N}+{ }_{a b} b \mathbb{N}$. Then, by Corollary 4.4 ,

$$
\mathrm{H}(\langle a, b\rangle, x)=\left(1-x^{a b}\right) \mathrm{H}\left(\mathbb{N}, x^{a}\right) \mathrm{H}\left(\mathbb{N}, x^{b}\right)=\frac{1-x^{a b}}{\left(1-x^{a}\right)\left(1-x^{b}\right)} .
$$

If we do this computation by using the formula

$$
\mathrm{H}(\langle a, b\rangle, x)=\frac{1}{1-x^{a}} \sum_{w \in \operatorname{Ap}(\langle a, b\rangle, a)} x^{w},
$$

we obtain

$$
\mathrm{H}(\langle a, b\rangle, x)=\frac{1}{1-x^{a}} \sum_{k=0}^{a-1} x^{k b}=\frac{1}{1-x^{a}} \frac{1-x^{a b}}{1-x^{b}} .
$$

Observe that this is a particular case of [21, Proposition 2] (see also [19, Theorem 4] for a relationship with inclusion-exclusion polynomials).

This idea can be generalized to any complete intersection affine semigroup. The base setting is the following.

Lemma 4.6. Let $A \subseteq \mathbb{N}^{m}$ be a set of linearly independent vectors. Then

$$
\mathrm{H}(\langle A\rangle, \mathbf{x})=\frac{1}{\prod_{\mathbf{a} \in A}\left(1-\mathbf{x}^{\mathbf{a}}\right)} .
$$

Proof. Assume that $A=\left\{\mathbf{a}_{1}, \ldots, \mathbf{a}_{k}\right\}$, and write $S=\langle A\rangle$. Notice that the map $\mathbb{N}^{k} \rightarrow S,\left(n_{1}, \ldots, n_{k}\right) \mapsto \sum_{i=1}^{k} n_{i} \mathbf{a}_{i}$ is a monoid isomorphism. Hence,

$$
\sum_{\mathbf{s} \in S} x^{s}=\sum_{n_{1} \in \mathbb{N}, \ldots, n_{k} \in \mathbb{N}} \mathbf{x}^{n_{1} \mathbf{a}_{1}+\cdots+n_{k} \mathbf{a}_{k}}=\prod_{i=1}^{k} \sum_{n \in \mathbb{N}}\left(x^{\mathbf{a}_{i}}\right)^{n},
$$

and the proof follows easily.

Proposition 4.7. Let $S$ be a free affine semigroup. Assume that $S=\left(\cdots\left(\left\langle\mathbf{v}_{1}, \ldots, \mathbf{v}_{e}\right\rangle+\theta_{e+1} \mathbf{v}_{e+1}\left\langle\mathbf{v}_{e+1}\right\rangle\right)+{ }_{\theta_{e+2} \mathbf{v}_{e+2}} \cdots\right)+_{\theta_{e+h} \mathbf{v}_{e+h}}\left\langle\mathbf{v}_{e+h}\right\rangle$. 
Then,

$$
\mathrm{H}(S, \mathbf{x})=\frac{\prod_{i=1}^{h}\left(1-\mathbf{x}^{\theta_{e+i} \mathbf{v}_{e+i}}\right)}{\prod_{i=1}^{e+h}\left(1-\mathbf{x}^{\mathbf{v}_{i}}\right)} .
$$

This is indeed a particular case of the following theorem.

Theorem 4.8. Let $S$ be a complete intersection affine semigroup minimally generated by $A$. Let $\mathbf{d}_{1}, \ldots, \mathbf{d}_{t-1}$ be as in Remark 3.5 ,

$$
\mathrm{H}(S, \mathbf{x})=\frac{\prod_{i=1}^{t-1}\left(1-\mathbf{x}^{\mathbf{d}_{i}}\right)}{\prod_{\mathbf{a} \in A}\left(1-\mathbf{x}^{\mathbf{a}}\right)} .
$$

If $S$ is a complete intersection, then any minimal system of generators $\left\{f_{1}, \ldots, f_{t-1}\right\}$ of the toric ideal associated to $S$ forms a regular sequence. With the same notation as in our Remark 3.5, Betti $(S)=$ $\left\{d_{1}, \ldots, d_{t-1}\right\}$. So, we may assume that $\operatorname{deg}_{S}\left(f_{i}\right)=d_{i}, i \in\{1, \ldots, t-1\}$ (repetitions may occur). Now, Theorem 4.8 also follows from [17, Exercise 5].

Remark 4.9. Observe that, if we subtract the degree of the numerator and denominator of the formula given in Theorem 4.8, we obtain formula (3.2).

Example 4.10. Let $S=\langle 4,5,6\rangle=\langle 4,6\rangle+{ }_{10} 5 \mathbb{N}=\left(4 \mathbb{N}+{ }_{12} 6 \mathbb{N}\right)+{ }_{10} 5 \mathbb{N}$. Then

$$
\mathrm{H}(\langle 4,5,6\rangle, x)=\frac{\left(1-x^{10}\right)\left(1-x^{12}\right)}{\left(1-x^{4}\right)\left(1-x^{5}\right)\left(1-x^{6}\right)} .
$$

The Frobenius number of $S$ is $10+12-(4+5+6)=7$.

Acknowledgments. Part of this research was performed while the second author visited the Université d'Angers, and he wants to thank the Département de Mathématiques of this university for its kind hospitality.

The authors would also like to thank the referees for their comments and suggestions. 


\section{REFERENCES}

1. J. Amos, I. Pascu, V. Ponomarenko, E. Treviño and Y. Zhang, The multidimensional Frobenius problem, Involve 4 (2011), 187-197.

2. F. Arslan, P. Mete and M. Şahin, Gluings and Hilbert functions of monomial curves, Proc. Amer. Math. Soc. 137 (2009), 2225-2232.

3. A. Assi, The Frobenius vector of a free affine semigroup, J. Alg. Appl. 11 (2012), 1250065, 10 pages.

4. A. Assi and P.A. García-Sánchez, Constructing the set of complete intersection numerical semigroups with a given Frobenius number, Appl. Alg. Eng. Comm. Comp. 24, (2013), 133-148.

5. V. Barucci, R. Fröberg and M. Şahin, On free resolutions of some semigroup rings, J. Pure Appl. Alg. 218 (2014), 1107-1116.

6. A. Barvinok and K. Woods, Short rational generating functions for lattice point problems, J. Amer. Math. Soc. 16 (2003), 957-979.

7. M. Bullejos and P.A. García-Sánchez, Minimal presentations for monoids with the ascending chain condition on principal ideals, Semigroup Forum 85 (2012), $185-190$.

8. S.T. Chapman, P.A. García-Sánchez, D. Llena, A. Malyshev and D. Steinberg, On the Delta set and the Betti elements of a BF-monoid, Arab. J. Math. 1 (2012), $53-61$.

9. S.T. Chapman, P.A. García-Sánchez, D. Llena, V. Ponomarenko and J.C. Rosales, The catenary and tame degree in finitely generated commutative cancellative monoids, Manuscr. Math. 120 (2006), 253-264.

10. M. D'Anna, V. Micale and A. Sammartano, Classes of complete intersection numerical semigroups, Semigroup Forum 88 (2014), 453-467.

11. C. Delorme, Sous-monö̈des d'intersection complète de $\mathbb{N}$, Ann. Sci. Ecole Norm. 9 (1976), 145-154.

12. K.G. Fischer, W. Morris and J. Shapiro, Affine semigroup rings that are complete intersections, Proc. Amer. Math. Soc. 125 (1997), 3137-3145.

13. P.A. García-Sánchez and M.J. Leamer, Huneke-Wiegand conjecture for complete intersection numerical semigroup rings, J. Alg. 391 (2013), 114-124.

14. P.A. García-Sánchez and I. Ojeda, Uniquely presented finitely generated commutative monoids, Pac. J. Math. 248 (2010), 91-105.

15. P.A. García-Sánchez, I. Ojeda and J.C. Rosales, Affine semigroups having a unique Betti element, J. Alg. Appl. 12 (2013), 1250177, 11 pages.

16. R. Jafari and S. Zarzuela Armengou, On monomial curves obtained by gluing, Semigroup Forum 88 (2014), 397-416.

17. M. Kreuzer and L. Robbiano, Computational commutative algebra 2, Springer-Verlag, Berlin, 2005.

18. N.J. Michelacakis and A. Thoma, On the geometry of complete intersection toric varieties, Arch. Math. 87 (2006), 113-123. 
19. P. Moore, Numerical semigroups, cyclotomic polynomials and Bernoulli numbers, Amer. Math. Month. 121 (2014), 890-902.

20. H. Nari, Symmetries on almost symmetric numerical semigroups, Semigroup Forum 86 (2013), 140-154.

21. J.L. Ramírez Alfonsín and Ø.J. Rødseth, Numerical semigroups: Apéry sets and Hilbert series, Semigroup Forum 79 (2009), 323-340.

22. J.C. Rosales, On presentations of subsemigroups of $\mathbb{N}^{n}$, Semigroup Forum 55 (1997), 152-159.

23. J.C. Rosales and P.A. García-Sánchez, On complete intersection affine semigroups, Comm. Alg. 23 (1995), 5395-5412.

24. J.C. Rosales and P.A. García-Sánchez, On Cohen-Macaulay and Gorenstein simplicial affine semigroups, Proc. Edinb. Math. Soc. 41 (1998), 517-537.

25. Finitely generated commutative monoids, Nova Science Publishers, Inc., Commack, NY, 1999.

26. , Numerical semigroups, Dev. Math. 20, Springer, New York, 2009.

Université D'Angers, DÉpArtement de Mathématiques, LAREMA, UMR 6093, 2 BD LAVoisier, 49045 Angers Cedex 01, France

Email address: assi@univ-angers.fr

Departamento de Álgebra, Universidad de Granada, E-18071 Granada, ESPAÑA

Email address: pedro@ugr.es

Departamento de Matemáticas, Universidad de Extremadura, E-06071 BadaJOZ, EsPAÑA

Email address: ojedamc@unex.es 